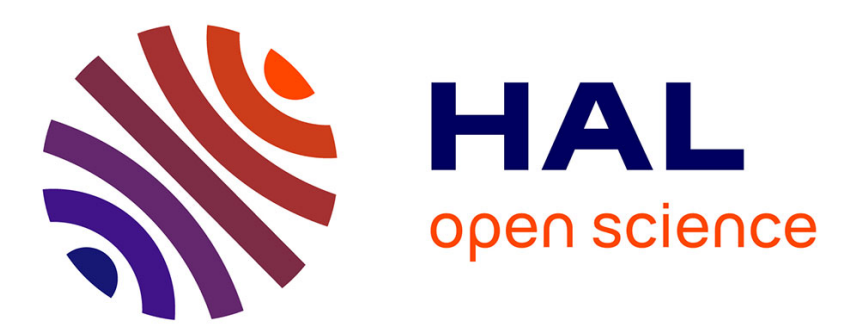

\title{
About lacunarity, some links between fractal and integral geometry, and an application to texture segmentation
}

Jacques Lévy Véhel

\section{To cite this version:}

Jacques Lévy Véhel. About lacunarity, some links between fractal and integral geometry, and an application to texture segmentation. [Research Report] RR-1188, INRIA. 1990. inria-00075371

\section{HAL Id: inria-00075371 \\ https://hal.inria.fr/inria-00075371}

Submitted on 24 May 2006

HAL is a multi-disciplinary open access archive for the deposit and dissemination of scientific research documents, whether they are published or not. The documents may come from teaching and research institutions in France or abroad, or from public or private research centers.
L'archive ouverte pluridisciplinaire HAL, est destinée au dépôt et à la diffusion de documents scientifiques de niveau recherche, publiés ou non, émanant des établissements d'enseignement et de recherche français ou étrangers, des laboratoires publics ou privés. 


\section{Rapports de Recherche}

64.

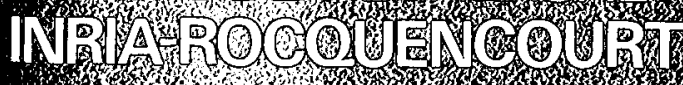

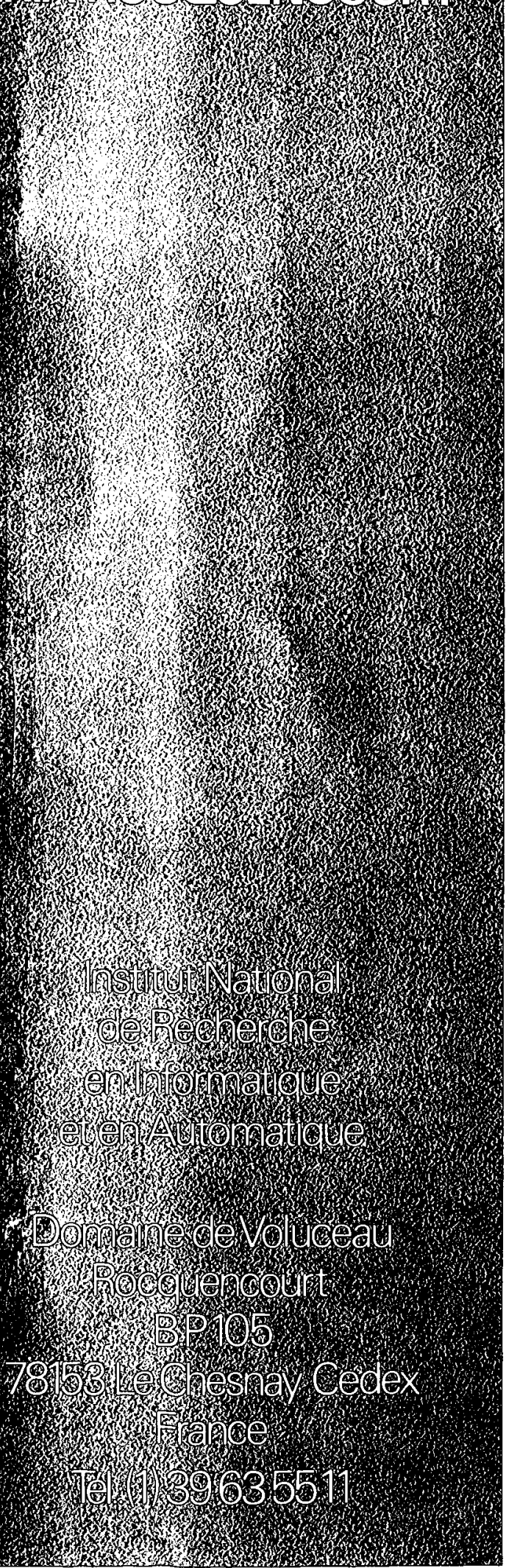

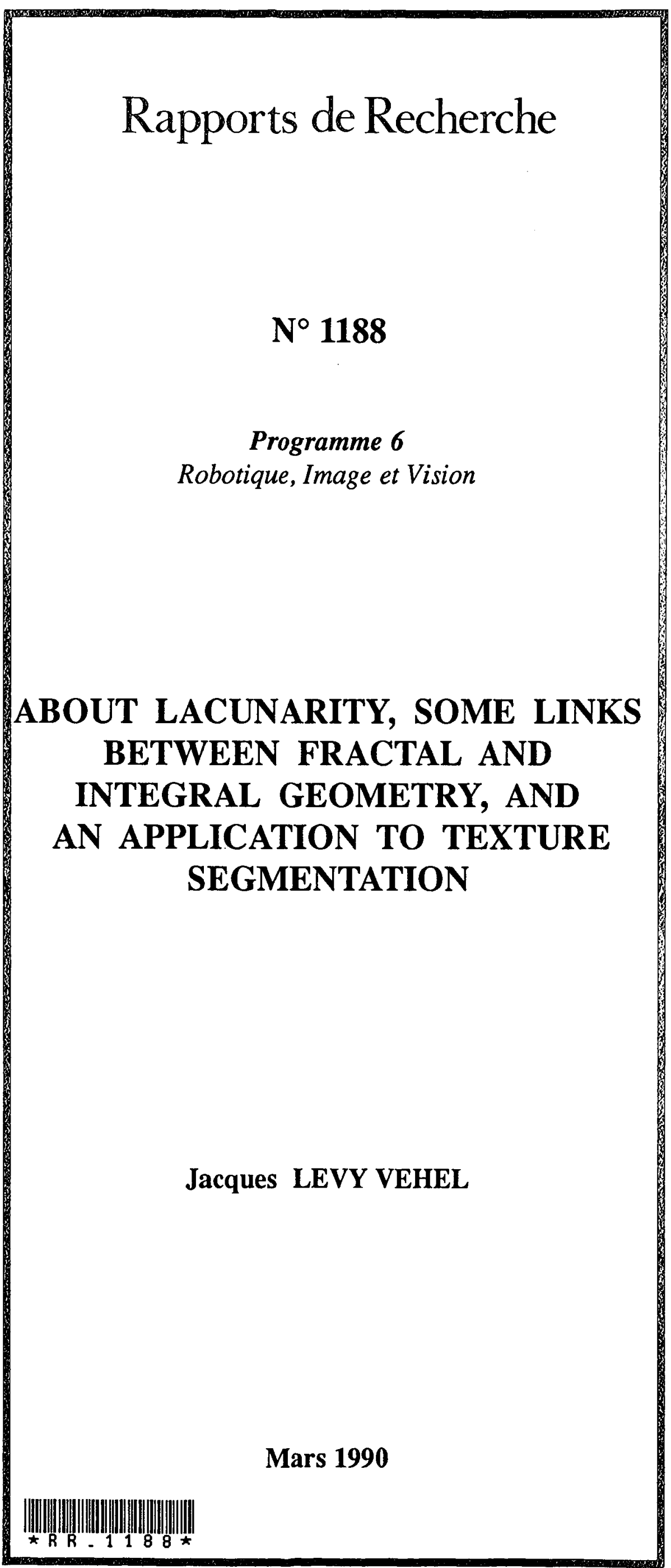




\title{
About Lacunarity, some links between Fractal and Integral Geometry, and an application to Texture Segmentation
}

\author{
A propos de Lacunarité, de relations entre la Géométrie Fractale et la Géométrie \\ Intégrale, et une application à la Segmentation de Textures
}

\section{Jacques LEVY VEHEL}

\author{
INRIA \\ Domaine de Voluceau \\ Rocquencourt - B.P. 105 \\ 78153 Le Chesnay Cédex \\ FRANCE
}

\begin{abstract}
In this work, we apply two techniques for segmentation of different states of one texture (e.g. deformations of an homogeneous texture) :

- Fractal Geometry, that deals with the analysis of complex irregular shapes which cannot well be described by the classical Euclidean geometry.

- Integral Geometry, that treats sets globally and allows to introduce robust measures.

We focus on the study of two parameters, lacunarity and Favard length, and proove a theoritical link between them.

As an application, we are able to achieve automatic classification of Lung diseases on the basis on SPECT images.

Dans ce papier, nous appliquons deux techniques pour la segmentation de différents états d'une texture (c'est-à-dire la déformation d'une texture homogène) :

- La Géométrie Fractale, qui permet d'analyser des formes complexes mal décrites par la géométrie euclidienne classique.

- La Géométrie Intégrale, qui traite les ensembles globalement, et permet d'introduire des mesures robustes.

Nous nous concentrons sur l'étude de deux paramètres, la lacunarité et la longueur de Favard, et nous prouvons un résultat théorique qui relie ces deux mesures.

Comme application, nous effectuons une classification automatique de maladies pulmonaires a partir d'images scintigraphiques.
\end{abstract}




\section{Introduction}

The aim of this work is to explore the possibility of automatic segmentation of different states of one texture. To explain what we mean by "different states of one texture", we give an example : let us consider SPECT images of the lung. For a normal lung, we obtain a certain texture T (see Figure 3). When a disease is present, $T$ varies locally: small and diffuse holes (for a chronic disease) or on the contrary one or two large holes (for a pulmonary embolism, Figure 4) appears on some places of $\mathrm{T}$, while others remain unchanged. Our interest lies then in measuring the "deformation" of an initially homogeneous texture.

Characteristic features of the images that we want to process are their complexity and the great amount of noise they contain. For instance, medical images (brain, lungs, heart, obtained through radiography, scintigraphy, echography) all give a great amount of information. These signals are difficult to process, mainly because they include a great number of regions and edges with a high degree of randomness and because the quality is not very good (low contrast, poor resolution..). The important information lies in the texture and not in the contours. Moreover, our will being to differentiate several states of one texture, classical methods of image analysis (regions or edges) may fail. That is why new techniques are needed to extract the interesting parameters.

Fractal Geometry, initially developped by Benoit Mandelbrot [Man77,Man82], can be of great help for the analysis of complex images, because it does not suppose that the studied objects have good properties of continuity; smoothness, etc.. On the contrary, fractal entities are irregular, rough, and in general can not be treated by Euclidean geometry: their main characteristic is that their complexity is the same whatever the level of analysis is. In particuliar, they have no derivatives. If such objects are considered as 'monsters' by classical geometry, they are often encountered in the real 3-D world: clouds, mountains, plants, textures can be treated as fractals [Vos85]. Physics and biology also include fractals. The main interest of Fractal Geometry is that it gives a way to quantify irregularity, and so allows to differentiate between two seemingly chaotic images.

Integral Geometry is another useful theory for processing our images : in this theory, classical measures such as length, perimeter, surface, are made on sets of the space; it also provides many useful exact or probabilistic results concerning intersections between sets. The interest for us lies in the fact that the technics used for the computing of the parameters are fairly insensitive to noise, because they use global knowledge and properties of the sets.

In this work we try to demonstrate the interest of the two mentionned theories for the processing of complex images. More precisely, we have chosen to study two parameters, the Favard length $f$ and the lacunarity $l$, and we have applied them to the problem of the classification of lung diseases. We also proove a theoritical result that links $f$ and $l$ in a simple way.

\section{$2 \quad$ Fractals}

To make it easy to understand the basic ideas of fractals and infinite level of complexity, a classic example is to try to measure the length of a coast, or a borderline between countries. If we use a rule of length $m$, and place it $n$ times along the coast to cover it, the computed length will be:

$$
L(m)=n \times m
$$

But with the rule of length $m$, we have lost the measure of little details, those that are smaller than $m$ : if we now use a rule of length $m^{\prime}$ smaller than $m$, we shall find $L\left(m^{\prime}\right)$ with:

$$
L\left(m^{\prime}\right)>L(m)
$$


The problem is that for most coasts, the function $L(m)$ has no upper bound: when $m$ tends towards $0, L$ tends towards infinity. To solve this problem, we use the notion of fractal dimension. If we had to measure an area instead of a length, we would use a formula like:

$$
\text { area }=n \times m^{2}
$$

and for a volume:

$$
\text { volume }=n \times m^{3}
$$

For a fractal object, there exists a number $D$ such that:

$$
n \times m^{l}=0 \text { if } l>D
$$

and

$$
n \times m^{l}=\infty \text { if } l<D
$$

$D$ is not an integer in the general case, it is called the fractal dimension. $D$ measures the degree of irregularity of the object. In the case of a curve, $D$ is always between 1 and 2. If $D$ is close to one, the curve will look like a 'normal' one (e.g. a piecewise smooth curve); if $D$ is close to 2 , the curve will be very complex in the sense that it would fill a large portion of the plane.

In general, the fractal dimension of a set $E$ is defined as follows: Let $\varepsilon$ be a real number, and consider spheres of diameter $\varepsilon$ in the $d$-dimensionnal space. Let $N(\varepsilon)$ be the number of such spheres needed to cover $E$. If:

$$
N(\varepsilon)=k\left(\frac{1}{\varepsilon}\right)^{D} \text { when } \varepsilon \rightarrow 0 ; \quad k \text { constant }
$$

then $D$ is called the fractal dimension of $E$. Practically, several methods can be used to compute $D$. As an example, we describe the box dimension method for a curve (see Figure 1). We first divide the plane into boxes of dimension $\varepsilon \times \varepsilon,(\varepsilon$ is large with respect to the dimension of the pixel) and compute the number $N(\varepsilon)$ of boxes that intersect the curve. We then use boxes of dimension $\frac{\varepsilon}{2} \times \frac{\varepsilon}{2}$, and compute the new $N\left(\frac{\varepsilon}{2}\right)$. We iterate until $\varepsilon$ reaches the size of one pixel. $D$ is given by the mean slope of the curve:

$$
\log N(\varepsilon) \text { versus } \log \left(\frac{1}{\varepsilon}\right)
$$

Many models have been proposed to perform a fractal analysis of natural phenomena. The well known fractionnal brownian motion, based on the analysis of the average absolute intensity difference of pixel pairs as a function of scale, allows to understand some statistically self-affine fractals as the result of random walks. However, our aim is not to build a model that fits in some sense a texture like șcintigraphic data of the lungs, but simply to recognize different states of one texture : in fact we are more interested in finding a set of parameters that performs such a segmentation.

Several authors have worked on the problem of fractal segmentation of textures. Pentland [Pen84] computes the Fourier transform of the image, finds the power spectrum, and estimate the fractal dimension as the mean slope of the function : $\log$ of the power spectrum versus log of the frequency. The fractal dimensions computed in two orthogonal directions gives a way to classify textures. Peleg [Peleg84] computes the size of the intensity surface area at different scales, determines the local slopes of $\log$ of area sizes versus $\log$ of scales, and is able to distinguish between six natural textures. Chen [Chen89] used fractionnal Brownian motion model to classify normal and abnormal ultrasonic liver images.

All these studies have in common the fact that they are founded on the use of the sole fractal dimension $D$. Some concentrate on computing a reliable value of $D$, others present a richer measure 

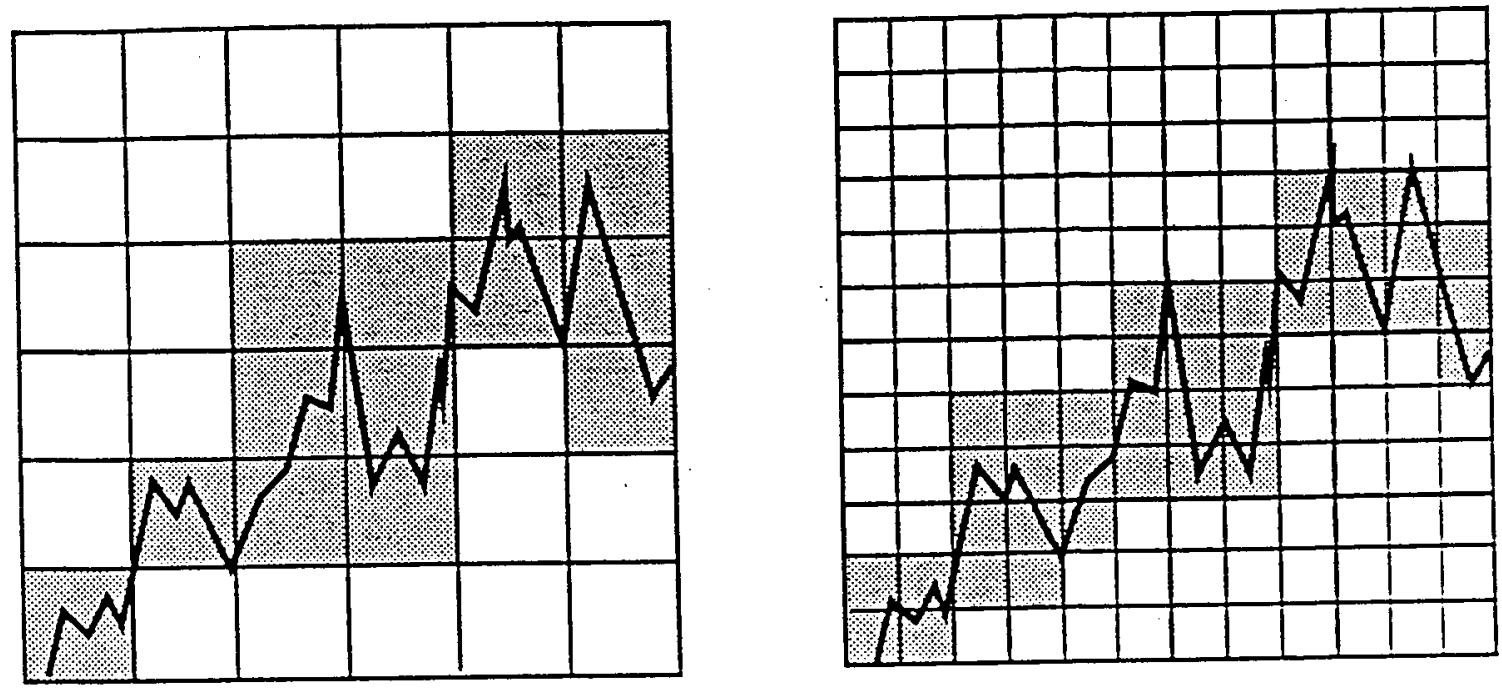

Figure 1: Computation of the fractal dimension with the boxes method

of roughness by including intermediate (in the sense of the degree of resolution) results. The new thing about our work is that we try to use also a second order fractal parameter for the segmentation which is totally independant from $D$.

Fractal dimension is of first order in the sense that it measures how the mass (or intensity) distribution varies with change of resolution. A second order parameter, the lacunarity, proposed by Mandelbrot [Man82], can be defined as follows : let $M$ be the total mass of the fractal object, and let $B$ be a window of given size $\varepsilon$ (for instance a disk of radius $\varepsilon$ ). One can compute the expected mass $m$ in $B$, and we know from fractal dimension $D$ that $m$ will vary as $\varepsilon^{D}$. As in statistics, we can build a second order parameter by measuring how far the actual mass $m^{\prime}$ in $B$ is from $m$. We then define lacunarity as :

$$
L=<\left(m^{\prime} / m-1\right)^{2}>
$$

Intuitively, $L$ will well measure the lacunarity of the object, e.g. the distribution of its gaps: a fractal is lacunar if its gaps are large (they include large discs).

Lacunarity is useful because it allows to distinguish two fractals that have same fractal dimension (see Mandelbrot [Man82]). Especially for us, it is a very interesting tool since, as a second order parameter, it measures the "distance" of a texture to homogeneity : that is what we want to quantify when we study the deformation of the texture.

\section{$3 \quad$ Integral Geometry}

Instead of making a general survey of Integral Geometry, which would be either too vast or too vague, we only introduce the notions that we are going to use. We first make the presentation in 


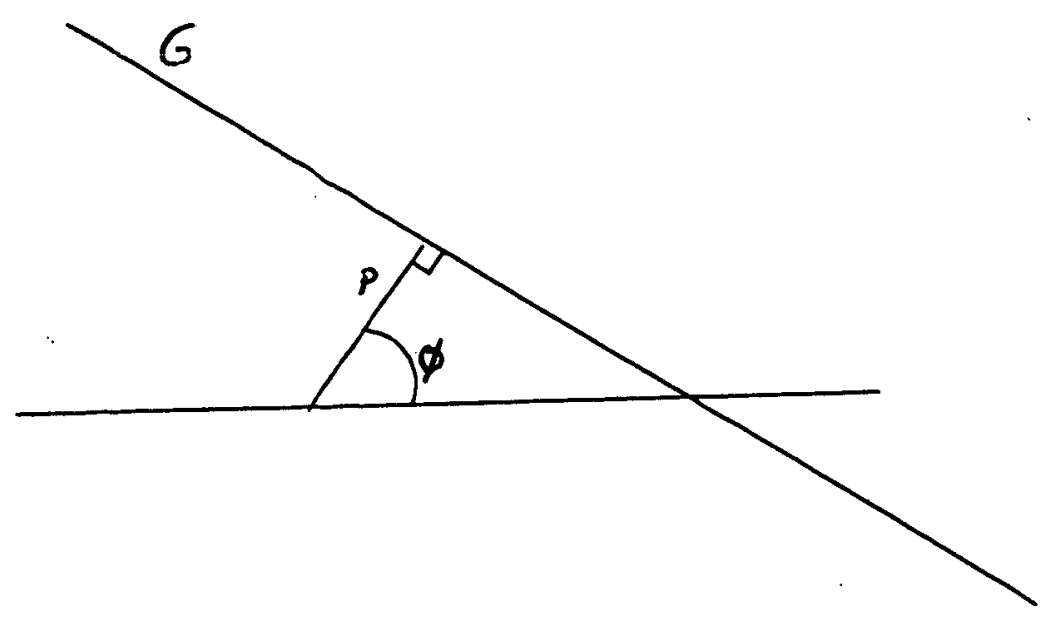

Figure 2: Definition of a line $G$

2-D, and then extend the results in 3-D.

Let $G$ be a straight line in the plane. We can determine $G$ by the angle $\phi$ that it makes with a fixed direction $(0 \leq \phi<2 \pi)$ and by its distance $p$ from a defined origine $O(p \geq 0)$. $(p, \phi)$ are the polar coordinates of the foot of the perpendicular from the origin onto the line (see Figure 2).

In order to extract desired parameters from the studied sets, we have to define a measure in the plane. More precisely, as we are going to work with lines, that is to use lines to measure sets, we must define the measure of a set of line. This measure must have some desirable properties, and first of all, must be invariant under motions. It is easy to show (see Santalo [San76] for this result and others in this chapter) that, up to a constant factor, the only invariant measure for a set of lines $G(p, \phi)$ is defined by the integral, over the set, of the differential form :

$$
d G=d p \wedge d \phi
$$

$d G$ is called the density for sets of lines.

This is an example of the use of $d G$ : let $K$ be a bounded convex set, and suppose that we want to measure the set of lines that intersect $K$. A simple computing gives :

$$
m(G ; G \cup K \neq \emptyset)=L
$$

where $L$ is the perimeter of $K$.

We find that the measure of the set of lines that intersect a bounded convex set is equal to its perimeter.

The result that we are going to use is the following one : Let $C$ be a piecewise differentiable curve, and for every line $G$ of the plane let $n(G)$ be the number of intersections between $G$ and 
$C(n \geq 0)$. Then :

$$
\int n(G) d G=2 L
$$

where $L$ is the length of $C$.

The very remarkable result is that this equality holds for any rectifiable curve, and can serve to actually measure lengths.

We can also extend the definition of length : let $E$ be a continuum of points in the plane, we define its Favard length as :

$$
f=\frac{1}{2} \int n(G) d G
$$

with the same notations as above. Note that $f$ can be infinite. This definition has the advantage that it can be applied to very complex objects (e.g. objects that cannot be viewed as simple curves) as the ones we encounter in medical images for example. Another very good property of $f$ is that it provides a simple, fast, and fairly precise way to compute numerically lengths, where other methods fail due to the complexity of the sets.

For the implementation, we have to discretize $d G$ : as for $p$, we define steps of one pixel, and it can be shown that choosing only eight directions for $\phi$ ensures that the error on $f$ will be lower that $8 \%$.

Up to now, we are able to measure curves, or 2-D contours. But scintigraphic images are grey level images, and therefore must be treated as 3-D shapes. The generalisation is simple : instead of computing lengths, we compute surfaces :

- we first cut the 3-D shape by a plane orthogonal to the $z$-axis, in order to obtain a 2-D curve,

- we compute the Favard length of this curve.

By doing this for several sucessive planes and adding all the lengths obtained, we can estimate fairly precisely the total "surface" of the shape. This measure has the great advantage of being really insensitive to any rotation or translation of the object. This is of great importance for comparing several medical images, where the conditions of acquisition are always fluctuating. Also, thanks to the integration along all directions and positions, $f$ is nicely insensitive to noise.

\section{$4 \quad$ Application}

We have applied our measures on different types of scintigrahic images of lungs. Three states have been considered: normal, chronic disease, pulmonary embolism. Even though it is easy for an experienced pratician to distinguish these three states on scintigraphic images, there are several reason why it is not obvious to do so for a computer:

- due to the nature of both the images and the diseases, only texture criteria can be helpful.

- the images are small, sometimes $128 \times 128$, often $64 \times 64$.

- they contain a great deal of noise.

- no general theory is available on the formation of the image or of the noise.

Our first aim is then to make an automatic classification of these diseases. A second and more ambitious one is to go further and help the pratician to: 

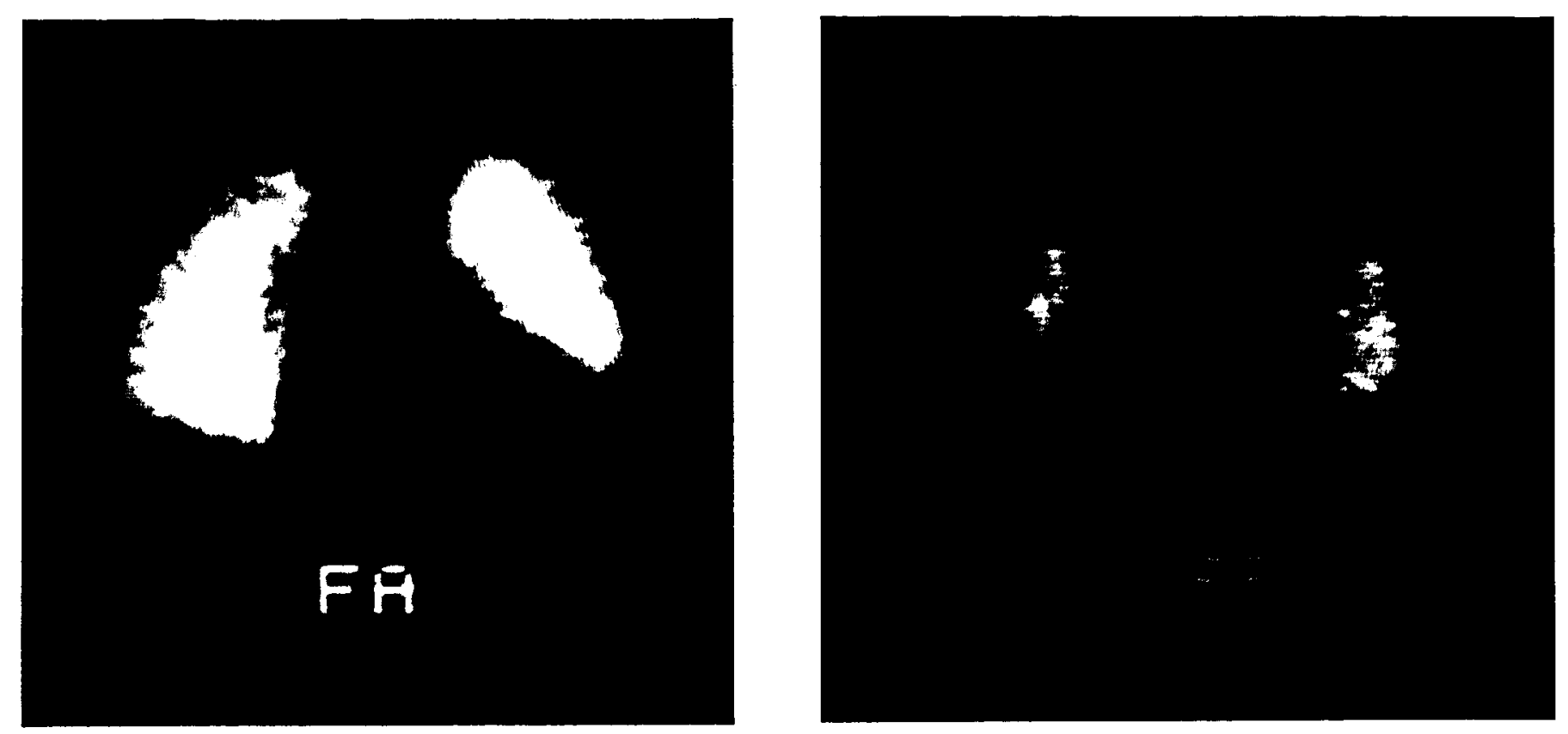

Figure 3: SPECT image of a normal lung

9
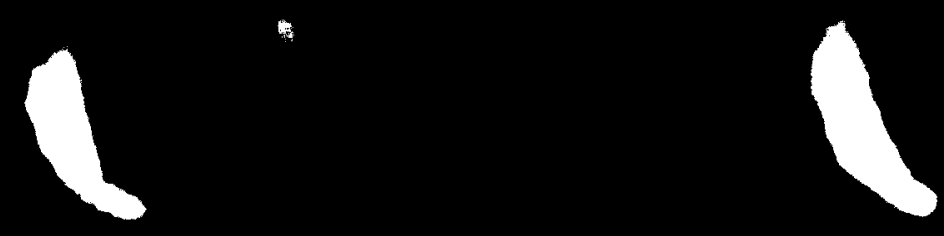

Figure 4: SPECT image of a pulmonary embolism 
- measure the progress of a chronic disease.

- detect a mix of chronic disease and pulmonary embolism.

These two tasks seems to be not easy to do visually, even for a pratician.

The interest of an automatic classification is that it would free the pratician of the task of analysing the scintigraphic images, saving him much time, since about one hundred of such images have to be processed every week in an average parisian hospital.

The set of data was composed of :

- nine 3-D images of projections, among which 3 chronic diseases $(c d 1, c d 2, c d 3) 3$ pulmonary embolism ( $p e 1, p e 2, p e 3)$ and 3 normal lungs $(n l 1, n l 2, n l 3$ ), (see Figure 23 , for SPECT images of lungs). Each "image" is $128 \times 128$, and contains around 20 projections,

- the nine corresponding images of slices, (reconstructed images) which are $64 \times 64$, and contain each around 16 slices.

These data all come from Hôpital Cochin, Paris.

The three parameters $(D, l, f)$ have been computed on each projection and each slice for each image. The set of the obtained values is too important for a practical use. Instead, we have computed the median value of each parameter for each image (projections and slices). We have found that the median value is better than the mean because some slices or projections (particularly extremal ones) contain too much noise with respect to the interesting signal, and so give weird results.

\subsection{Fractal analysis}

The computation of $D$ has to goals:

- When we compute $D$ on a grey level image, considered as a 3-D shape, we always find a number between 2 and 3 .

If we obtain $D=2$, it means that the studied surface is not a fractal, and that further analysis is meaningless. This implies that we first need to compute $D$ to validate our approach. In our special case of several slices or projections in one image, we can also check that $D$ remains approximatively the same for each sub-image, so that we can speak of the fractal dimension of a particular lung.

- If $D$ is strictly greater than 2 , we can see if there are significant differences between the $D$ of the three types of lungs.

Leaving apart extremal sub-images, we have obtained the remarkable result that all slices and all projections yield a $D$ of 2.3 , regardless of the type of lung (see Figure 5).

In fact, $D$ varies between 2.2 and 2.4 , but we must keep in mind the small size of the images that does not allow a good precision on $D$, certainly less than 0.1 , and so the differences are not significant.

This result confirms that SPECT images of the lungs are fractal, but shows that $D$ fails to separate the diseases.

When we compute $l$, we again find that, for a large number of slices or projections of one image, the lacunarity remains approximatively constant. But now, $l$ changes from one image to another: for the normal lungs, $l$ is typically 0.2 ; for the chronic diseases, it is 0.6 , and for pulmonary embolism, it is 0.8 . These normalised values are computed on the projections.

They show that actual separation of the three types of lungs is possible with respect to $l$. 


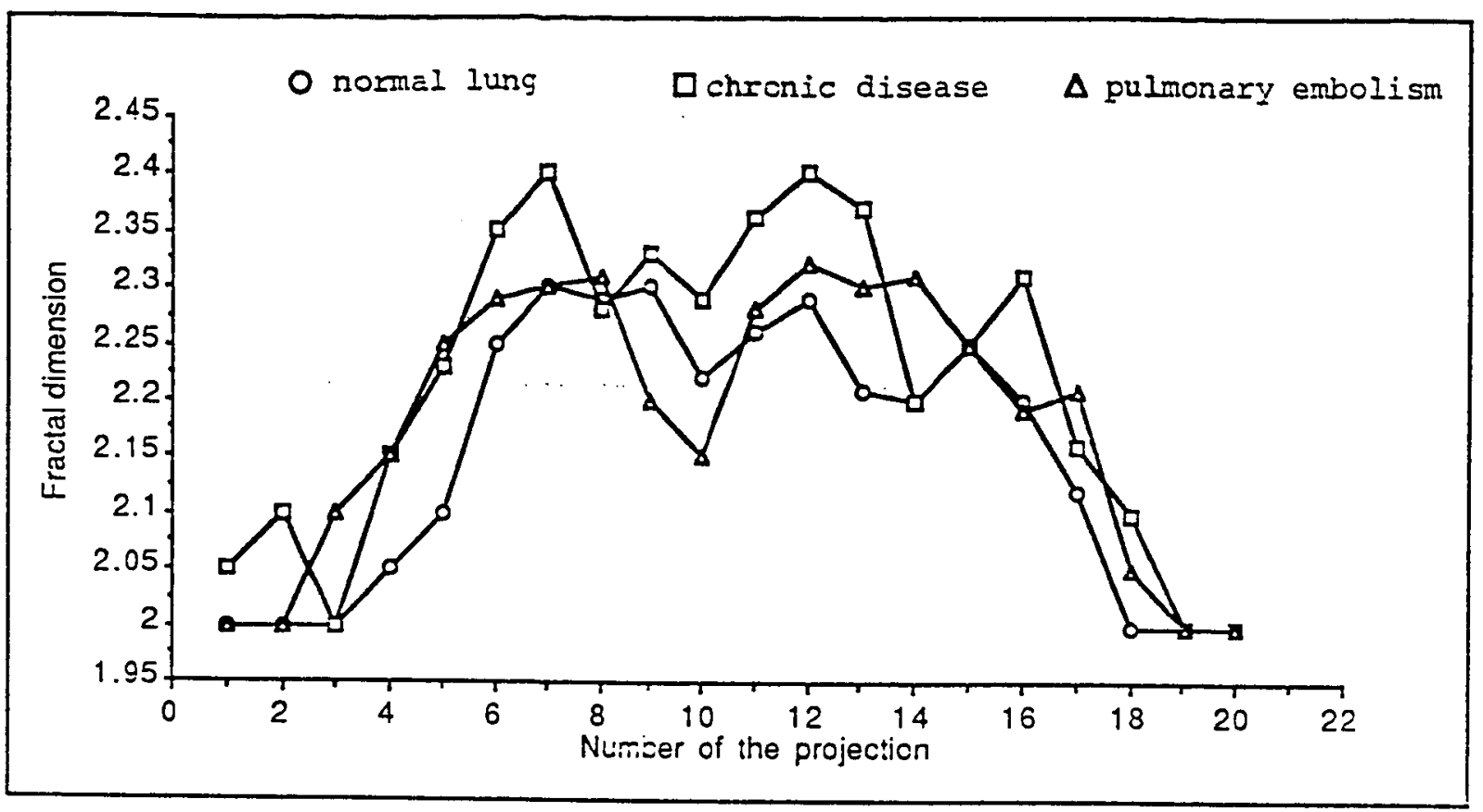

Figure 5: Typical values of $D$ for normal lung, chronic disease, pulmonary embolism

\subsection{Integral geometry analysis}

The computing of $f$ for projections gives (normalised values) :

- for normal lung, $f$ is typically 0.9 ,

- for chronic diseases, $f$ is typically 0.7 ,

- for pulmonary embolism, $f$ is typically 0.4 .

There again, we find that $f$ can separate the three types.

On Table 1 are given the median values of $l$ and $f$ for each projection and slice image: we can see that for projections, the segmentation works allright. But for the slices, there is an inversion between pel and $c d 3$. Several factors explain this error:

- slice images are reconstructed ones, and so contains more noise than projection images,

- slice images are only $64 \times 64$ and so the computing of $F$ and $L$ is less precise.

- the pulmonary embolism on image pel is not easy to see, because is is small and appears only on a few slices. This makes pe 3 a difficult image to analyse.

\section{Theoritical hints}

All our measures (on natural and computed images) have shown a sort of correlation between $f$ and $l$. More precisely, for two fractals $F_{1}, F_{2}$ with same dimension and mass, we found that: 


\begin{tabular}{|c|c|c|c|c|}
\hline & $l$ for projections & $l$ for slices & $f$ for projections & $f$ for slices \\
\hline nl1 & 0.18 & 0.17 & 0.91 & 0.9 \\
\hline n12 & 0.20 & 0.21 & 0.9 & 0.88 \\
\hline n13 & 0.21 & 0.23 & 0.9 & 0.87 \\
\hline cd1 & 0.55 & 0.53 & 0.73 & 0.7 \\
\hline cd2 & 0.62 & 0.64 & 0.67 & 0.65 \\
\hline cd3 & 0.63 & 0.69 & 0.65 & 0.5 \\
\hline pe1 & 0.79 & 0.68 & 0.48 & 0.52 \\
\hline pe2 & 0.80 & 0.78 & 0.41 & 0.4 \\
\hline pe3 & 0.83 & 0.82 & 0.35 & 0.35 \\
\hline
\end{tabular}

Table 1:

$$
\left(f\left(F_{1}\right)<f\left(F_{2}\right)\right) \quad \text { equivalent to } \quad\left(l\left(F_{1}\right)>l\left(F_{2}\right)\right)
$$

Intuitively, it is easy to understand that, if we change the frontier of a set $E$ in such a way that:

- the mass remains constant,

- the fractal dimension remains constant,

- we add sinuosities to the contour,

then the Favard length will increase ("the perimeter" of the set is higher), and the lacunarity will decrease because the set and its complementary will be mixed in a more intricate way and the homogeneity of the mass distribution will be greater.

To confirm theoritically these results, we have tried and dig deeper in the definition of $l$.

Our aim is to find a first order approximation of $l$ when the size of the window goes to 0 . The hypothesis and notations are :

Let $D$ be a disk of fixed radius $D_{R}$.

Let $C_{1}, C_{2}, \ldots, C_{n}$ be $\mathrm{n}$ closed piecewise smooth curves included in the interior of $D$.

Let $E$ be the set composed of the union of all the interiors of the $C_{i}$, and $E$ be the complementary of $\underline{E}$ in $D$.

Let $S$ be the area of $\mathrm{D}, \alpha S$ the area of $E$, and then $(1-\alpha) S$ the area of $\underline{E}$. $E$ represent the set we want to measure, and $\underline{E}$ the "holes" in the texture.

Let $P$ be the length of the frontier of $E$.

Let $r$ be the radius of the window in which we compute the actual mass. $r$ is close to 0 .

Let us consider a saussage of width $2 r$ around the frontier of $E$ (see Figure 6). This saussage defines three regions in $D$ (if $r$ is small enough, since the $C_{i}$ are in the interior of $D$, the saussage is also included in $D$ ) :

- $R_{1}$ : the points of $D$ outside the saussage and included in $E$. The aera of $R_{1}$ is $\alpha S-P r$.

- $R_{2}$ : the points of $D$ outside the saussage and outside $E$. The area of $R_{2}$ is $(1-\alpha) S-P r$.

- $R_{3}$ : the points of the saussage.

We recall that :

$$
l^{2}=\iint_{P}\left(\frac{\iint_{B(r, x, y)} 1_{E} d r d \theta}{<m>}-1\right)^{2} d x d y
$$

where : 


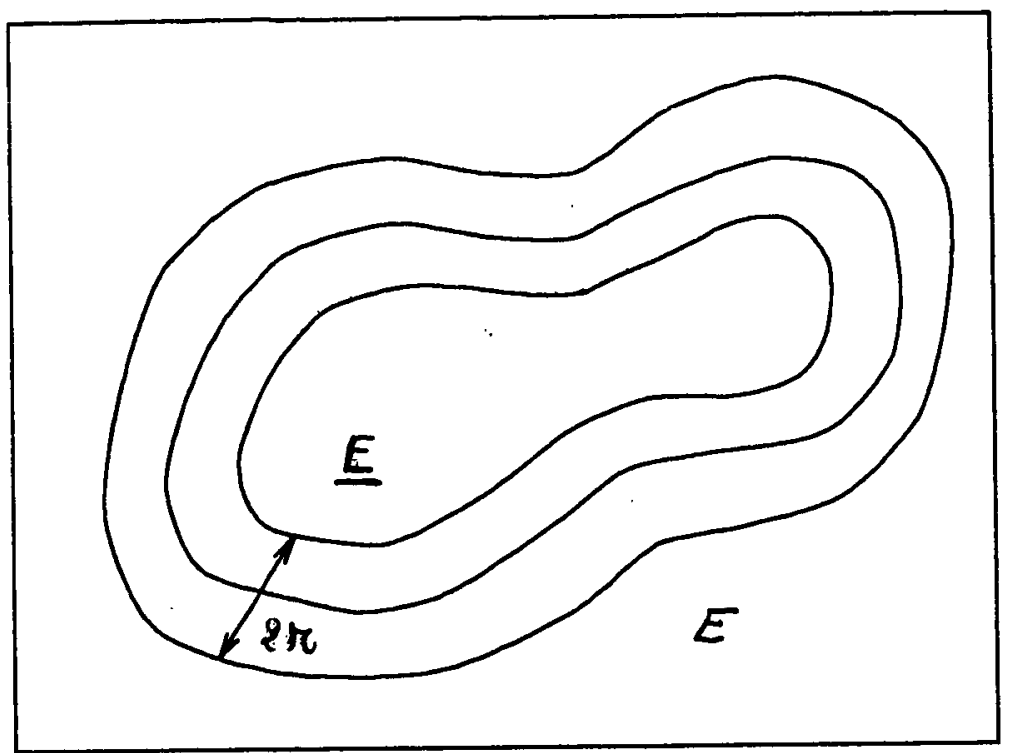

Figure 6: Definition of the three regions

- $B$ is the disk of radius $r$ centered on $(x, y)$

- $\langle m\rangle$ is the average mass :

$$
<m>=\frac{\iint_{D} \mathbf{1}_{E} d x d y}{\iint_{D} \mathbf{1}_{D} d x d y} \iint_{B(r)} d x d y
$$

It is possible to re-write the definition of $l$ using $f_{r}(x, y)=$ proportion of "mass" in $B(r, x, y)$. Then the actual mass in $B$ is : $\pi r^{2} \times f_{r}(x, y)$ and :

$$
l^{2}=\frac{1}{S} \iint\left(\frac{f_{r}(x, y)}{\alpha}-1\right)^{2} d x d y
$$

We split the integral on $D$ in three terms :

- $l_{1}^{2}=\frac{1}{\mathrm{~s}} \iint_{\mathrm{R}_{1}}\left(\frac{\mathrm{f}_{\mathrm{r}}(\mathrm{x}, \mathrm{y})}{\alpha}-1\right)^{2} \mathrm{dxdy}$ in $R_{1}, f_{r}(x, y)=1$ everywhere :

$$
l_{1}^{2}=\frac{1}{S} \iint_{R_{1}}\left(\frac{1}{\alpha}-1\right)^{2} d x d y=\frac{1}{S}\left(\frac{1}{\alpha}-1\right)^{2} \times(\alpha S-P r)
$$

- $\mathrm{l}_{2}^{2}=\frac{1}{\mathrm{~S}} \iint_{\mathrm{R}_{2}}\left(\frac{\mathrm{f}_{\mathrm{r}}(\mathrm{x}, \mathrm{y})}{\alpha}-1\right)^{2} \mathrm{dxdy}$

in $R_{2}, f_{r}(x, y)=0$ everywhere : 


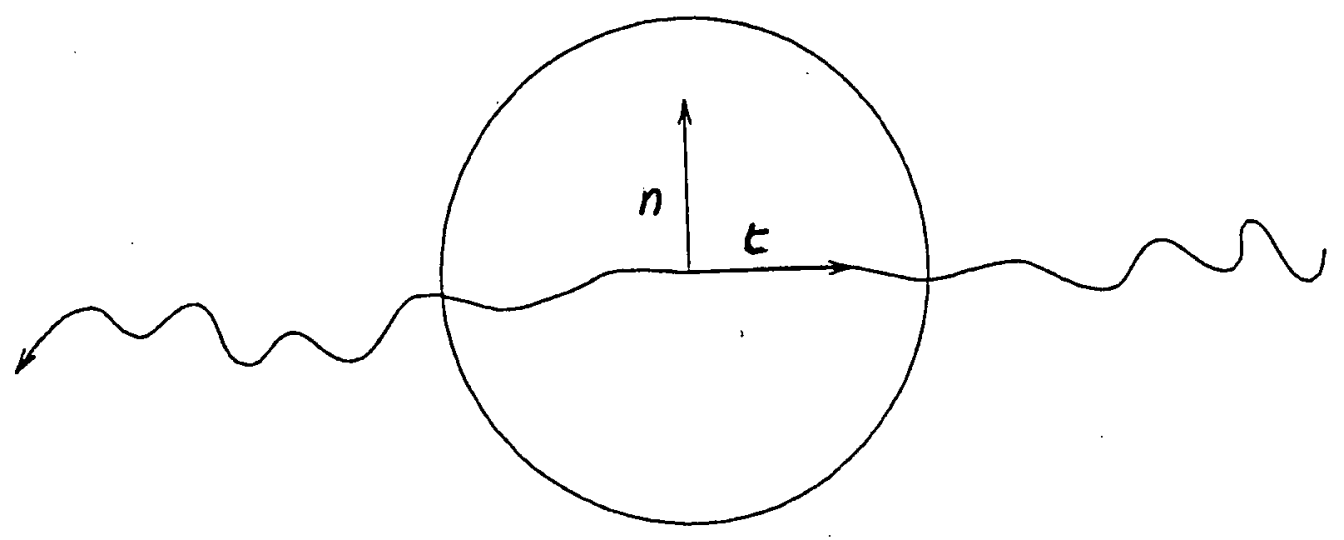

Figure 7:

$$
l_{2}^{2}=\frac{1}{S} \iint_{R_{2}} d x d y=\frac{1}{S}((1-\alpha) S-P r)
$$

- $l_{3}^{2}=\frac{1}{\mathrm{~S}} \iint_{\mathrm{R}_{3}}\left(\frac{\mathrm{f}_{\mathrm{r}}(\mathrm{x}, \mathrm{y})}{\alpha}-1\right)^{2} \mathrm{dxdy}$.

We do the change of variables:

$$
(x, y) \longmapsto(n, t) \quad \Phi_{r}(n, t)=f_{r}(x, y) \quad \text { and } \quad d x d y=d n d t
$$

(for each $(\mathrm{x}, \mathrm{y})$, the transformation is a rotation) where $n$ and $t$ are the radial and tangent lengths along the frontier of $E$ (they exist almost everywhere). If $r$ is small enough, we can almost everywhere consider locally the frontier as a part of a straight line. Under these conditions, the dependance on $t$ disappears since the proportion of full mass in $B$ does not change when we make a small tangent walk along the frontier (see Figure 7). In the end, if we integrate with respect to $t$ along the whole frontier of $E$, we shall have :

$$
\begin{aligned}
l_{3}^{2} & =\frac{1}{S} \iint_{R_{3}}\left(\frac{\Phi_{r}(n, t)}{\alpha}-1\right)^{2} d n d t \\
& =\frac{P}{S} \int_{R_{3}}\left(\frac{\Psi_{r}(n)}{\alpha}-1\right)^{2} d n
\end{aligned}
$$

with :

$$
\Psi_{r}(n)=\Phi_{r}(n, t)
$$




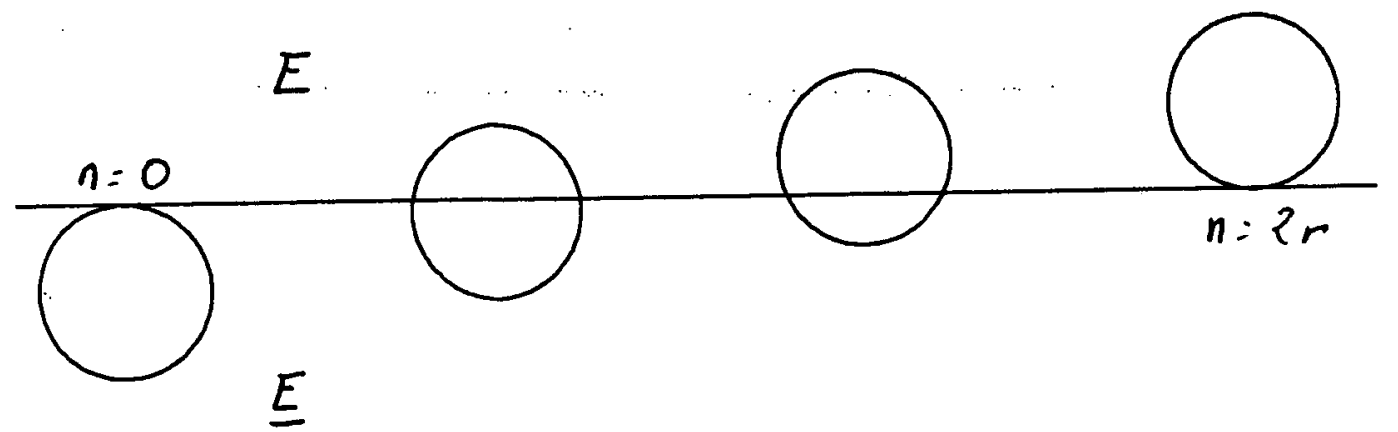

Figure 8:

The situation for this integral is the following one : we have a disk $B$ that walks orthogonally from the outside to the inside of $E$, starting from a position where the intersection between $B$ and $E$ is empty (for example the value of $n$ is 0 at this point) to a position where $B$ is included in $E$ $(n=2 r$, see Figure 8$)$. Finally, we can see that we have to integrate the areas of all the lunules from 0 to a full disk, Figure 9 ). We shall obtain a constant depending only on $r$. The computing of the area of one lunule gives :

$$
\sigma=\frac{r^{2}}{2}(\theta-\sin \theta)
$$

and :

$$
\Psi_{r}(n)=\frac{\sigma}{\pi r^{2}}=\frac{\theta-\sin \theta}{2 \pi}
$$

We still have to express $n$ as a function of $\theta$ :

$$
\begin{aligned}
n & =r\left(1-\cos \frac{\theta}{2}\right) \\
d n & =\frac{r}{2} \sin \frac{\theta}{2} d \theta
\end{aligned}
$$

then :

$$
l_{3}^{2}=\frac{P}{S} \int_{0}^{2 \pi}\left(\frac{\theta-\sin \theta}{2 \pi \alpha}-1\right)^{2} \frac{r}{2} \sin \frac{\theta}{2} d \theta
$$




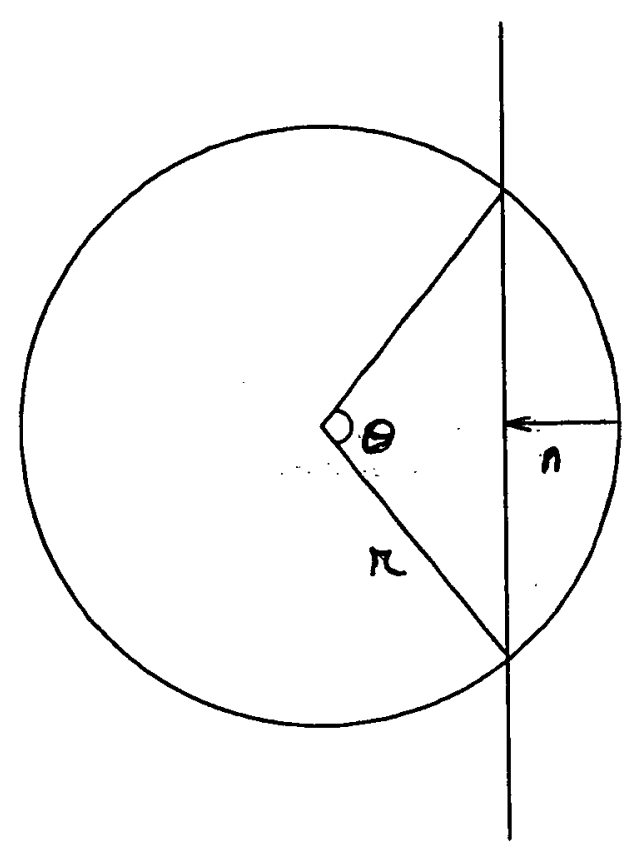

Figure 9:

$$
=\frac{\operatorname{Pr}}{\alpha^{2} S}\left(\frac{-128}{45 \pi^{2}}+\alpha^{2}+(1-\alpha)^{2}\right)
$$

In the end, we find for $l$ :

$$
\begin{gathered}
l^{2}=l_{1}^{2}+l_{2}^{2}+l_{3}^{2} \\
l^{2}=\frac{1-\alpha}{\alpha}-\frac{128}{45 \alpha^{2} \pi^{2}} \frac{P r}{S}
\end{gathered}
$$

Before we go further, let us pause and make some remarks about equality 2 :

- If the frontier of $E$ is a piecewise smooth curve of finite length, we obtain the limit:

$$
\lim _{r \rightarrow 0} l^{2}=l_{0}^{2}=\frac{1-\alpha}{\alpha}
$$

As expected, if $\alpha$ is high (near one) $l^{2}$ is near 0 , because the set $E$ will fill almost entirely the domain $D$. The limit when $\alpha$ goes down to 0 is more difficult to appreciate. Equality 2 gives: $l^{2} \rightarrow \infty$, but we must remember that, in the definition of $l$ (equality 1 ), the average mass $\langle m\rangle$ appears in the denominator.

However, when $r$ decreases till 0 , the limit value of $l^{2}$ only depends on $\alpha$, which seems reasonnable for a piecewise smooth curve of finite length.

Now let us move to the first order term. The variables are $P$ and $r$. 
- First, remark the negative sign of this term: as $r$ goes to $0, l^{2}$ increases to $l_{0}^{2}$, which is intuitive.

- For fixed $\alpha$ and $r, l^{2}$ is a decreasing function of $P$ : we find here the justification of our intuition and measure, that, everything else remaining constant, the lacunarity shall be low is the frontier of the set has many sinuosities, and high if it is straight.

- For a fixed perimeter, $l^{2}$ is also a decreasing function of $r$; this is also intuitive: at the limit of $E$ being included in a single $B(r, x, y)$, we shall have $m=<m>$ (notation of equality 1 ) and $l^{2}=0$. If $r$ is very small, then the $B(r, x, y)$ will be able to measure very small details and variations of mass, and $l^{2}$ will be high.

However, our real interest lies in measuring $l^{2}$ for fractals, and our whole procedure till now has been based on the property of the frontier of $E$ to be piecewise smooth and of finite length.

Let now the frontier of $E$ be a fractal. This means that at least one $C_{i}$ is a fractal. To find an extension to our formula, we are going to use the classical approximations of our set $E$ at different resolutions. Let $R(n)=2 n$, for $n$ in $\mathrm{N}^{*}$ be a sequence of increasing resolutions. We state the following definition :

$E_{n}$ is an approximation of $E$ at the resolution $R(n)$ iff :

- The frontier of $E_{n}$ is piecewise smooth.

- $d_{h}\left(E, E_{n}\right)<\frac{1}{R(n)}\left(d_{h}\right.$ is the Haussdorff distance between sets).

We can see that, if we increase the resolution to infinity, we can find a sequence of $E_{n}$ that goes to $E . E_{n}$ is an approximation of $E$ such that the only "details" that differ between $E$ and $E_{n}$ are "smaller" than $\frac{1}{R(n)}$.

For a fixed $n$, formula 2 holds and we can write $l$ as a function of $n$ :

$$
l^{2}(n)=\frac{1-\alpha(n)}{\alpha(n)}-\frac{128}{45 \alpha^{2}(n) \pi^{2}} \frac{f(n) r(n)}{S}
$$

(Clearly, $S$ do not depend on $\mathrm{n}$ )

- Instead of writing $P(n)$ we have written $f(n)$, that is the Favard length of $E_{n}$ : this is to gain some generality, and take into account special cases : now, we need only define the frontier of $E$ as a collection of continua of points.

We have to find out what happens when $\mathrm{n}$ changes :

When the resolution doubles, new details of $E$ will appear in $E_{n}$, of size between $\frac{1}{R(n)}$ and $\frac{1}{R(n-1)}$. All the new parts in $E_{n}$ that are not in $E_{n-1}$ are included in disks of diameter $\frac{1}{R(n-1)}-\frac{1}{R(n)}$. This implies the relation :

$$
r(n)=\frac{r_{0}}{n}
$$

where $r_{0}$ is a constant.

It is easy to see that the function $\alpha(n)$ goes to a limit $\alpha$ when $n$ goes to infinity

If we consider the whole frontier of $E$ as one fractal lumping all the $C_{i}$, we can measure its fractal dimension $D$ and we have by definition :

$$
f(n)=f_{0} \frac{1}{n^{1-D}}
$$


$f_{0}$ being a constant.

We can now write the lacunarity for a set whose frontier is fractal:

$$
l^{2}(n)=\frac{1-\alpha(n)}{\alpha(n)}-\frac{128}{45 S \alpha^{2}(n) \pi^{2}} \frac{f_{0} r_{0}}{n^{2-D}}
$$

Since $D$ is between 1 and 2 , we still have a finite limit for $l$.

It is important to note that $D$ is the fractal dimension of the whole frontier of $E$. Each indivudual $C_{i}$ may be (and in general is) of different dimension $D_{i}$

\section{Conclusion}

In this work, we have presented a new approach to the problem of the segmentation of different states of one texture, based on the use of both fractal and integral geometry parameters. The interest of these measures is that they are adapted to complex, irregular, noisy shapes. Moreover, we have prooved a result that binds $l$ and $f$ under certain assumption, and that help to understand the links between Fractal and Integral Geometry, which are not well known. As an application, we have found a way to make an automatic classification of certain lung diseases. Further developpement will hopefully allow to measure evolution of these diseases and simultaneous occurence of chronic disease and pulmonary embolism.

\section{Acknowledgements}

The author wishes to thank Jean-Marie Rocchisani for furnishing the whole set of SPECT images and Robert Ehrlich for many helpfull discussions. Many thanks also to Nathalie Gaudechoux.

\section{References}

\section{References}

[Chen89] C.C. Chen, J.S. Daponte, and M.D. Fox. Fractal feature analysis and classification in medical imaging. IEEE Transactions on Medical Imaging, 8(2):147-170, June 1989.

[Man77] B.B Mandelbrot. Fractals: Form, Chance and Dimension. CA: Freeman, San Fransisco, 1977.

[Man82] B.B Mandelbrot. The Fractal Geometry of Nature. CA: Freeman, San Fransisco, 1982.

[Pen84] A. Pentland. Fractal-based description of natural scenes. IEEE Transactions on Pattern Analysis and Machine Intelligence, PAMI-6:666-674, 1984.

[Peleg84] S. Peleg, J. Naor, R. Hartley, and D. Avnir. Multiple resolution texture analysis and classification. IEEE Transactions on Pattern Analysis and Machine Intelligence, PAMI6(4), July 1984.

[San76] L.A. Santalo. Integral Geometry and Geometric Probability. Addison-Wesley Publishing Company, 1976.

[Vos85] R.F. Voss. Random fractal forgeries. In R.A. Earnshaw, editor, Computer Graphics, Springer-verlag Berlin Heidelberg, 1985. 
